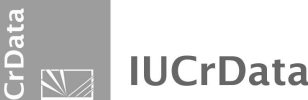

ISSN 2414-3146

Received 6 January 2017

Accepted 9 January 2017

Edited by H. Stoeckli-Evans, University of Neuchâtel, Switzerland

Keywords: crystal structure; chalcone; trans conformation; $\mathrm{C}-\mathrm{H} \cdots \pi$ interactions.

CCDC reference: 1526365

Structural data: full structural data are available from iucrdata.iucr.org

\section{(E)-1-(5-Chlorothiophen-2-yl)-3-(p-tolyl)prop-2-en- 1-one}

\author{
Karthik Kumara, ${ }^{a}$ S. Naveen, ${ }^{\text {b }}$ M. G. Prabhudeva, ${ }^{\text {c }}$ K. Ajay Kumar, ${ }^{\text {c N. K. Lokanath }}{ }^{\mathrm{a} *}$
} and Ismail Warad ${ }^{d_{*}}$

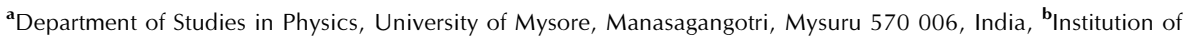
Excellence, University of Mysore, Manasagangotri, Mysuru 570 006, India, 'Department of Chemistry, Yuvaraja's College, University of Mysore, Mysuru 570 005, India, and d Department of Chemistry, Science College, An-Najah National University, PO Box 7, Nablus, West Bank, Palestinian Territories. *Correspondence e-mail:

lokanath@physics.uni-mysore.ac.in, khalil.i@najah.edu

In the title compound, $\mathrm{C}_{14} \mathrm{H}_{11} \mathrm{ClOS}$, the trans conformation of the $\mathrm{C}=\mathrm{C}$ double bond in the central enone group is confirmed by the $\mathrm{C}-\mathrm{C}=\mathrm{C}-\mathrm{C}$ torsion angle of $178.3(4)^{\circ}$. The molecule is non-planar as seen by the dihedral angle of $22.6(2)^{\circ}$ between the chlorothiophene and the $p$-toluene rings. In the crystal, molecules are linked by pairs of $\mathrm{C}-\mathrm{H} \cdots \pi$ interactions, forming inversion dimers. There are no other significant intermolecular interactions present.
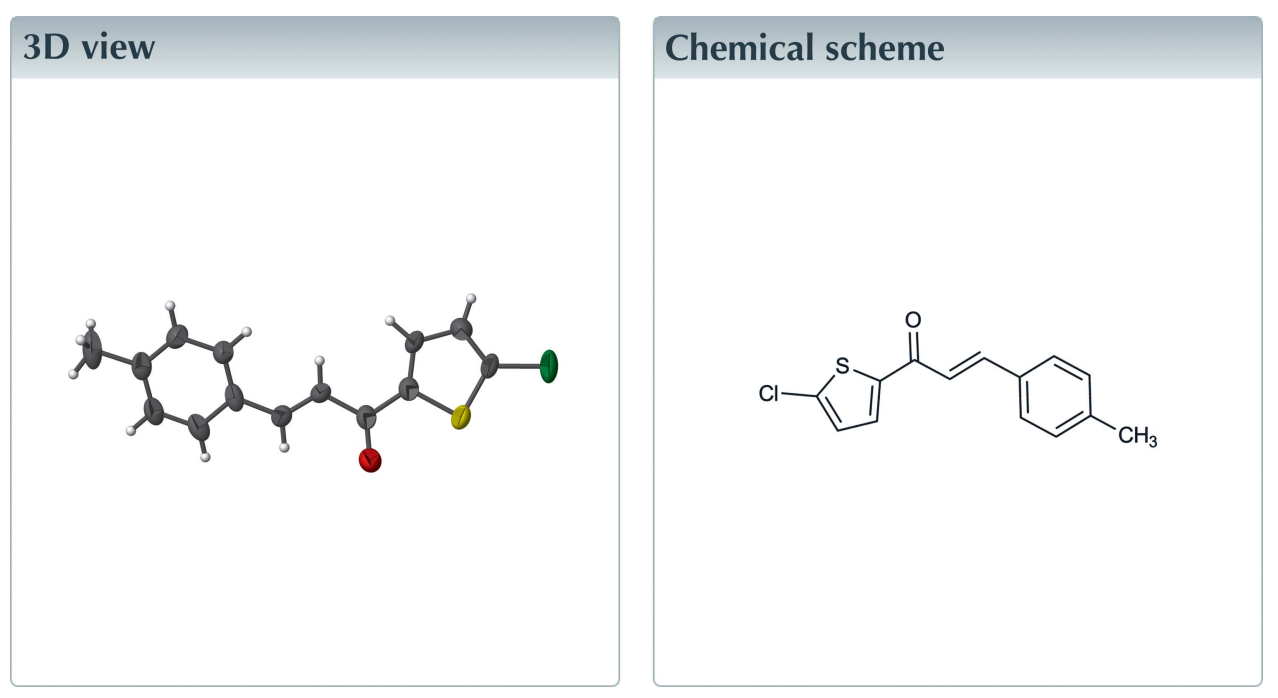

\section{Structure description}

The usual method for the synthesis of chalcones involves the condensation of an aromatic aldehyde and aromatic ketone in the presence of aqueous alkaline bases (Naveen et al., 2016a). Chalcones and their derivatives demonstrate a wide range of biological activities such as antidiabetic, antineoplastic, antitubercular, antiarrhythmic, hypnotic, antiangiogenic, antiprotozoal, antibacterial, antisteroidal, cardioprotective. In view of the broad spectrum of applications associated with chalcones and as a part of our ongoing work on such molecules (Tejkiran et al., 2016; Naveen et al., 2016b), we report herein the synthesis and crystal structure of the title compound.

The molecular structure of the title compound is shown in Fig. 1. The molecule is nonplanar, with a dihedral angle of $22.6(2)^{\circ}$ between the chlorothiophene and $p$-toluene rings that are bridged by the olefinic double bond. This value is in good agreement with the value of $19.13(15)^{\circ}$ reported earlier between the aromatic rings in the related chalcone derivative (E)-3-(2,3-dichlorophenyl)-1-(4-fluorophenyl)prop-2-en-1-one (Naveen et al., 2016b). The trans conformation about the $\mathrm{C} 6=\mathrm{C} 7$ double bond in the 
Table 1

Hydrogen-bond geometry $\left(\AA,^{\circ}\right)$.

$\mathrm{Cg}$ is the centroid of the $\mathrm{C} 8-\mathrm{C} 13$ ring.

\begin{tabular}{lllll}
\hline$D-\mathrm{H} \cdots A$ & $D-\mathrm{H}$ & $\mathrm{H} \cdots A$ & $D \cdots A$ & $D-\mathrm{H} \cdots A$ \\
\hline $\mathrm{C} 14-\mathrm{H} 14 A \cdots C g^{\mathrm{i}}$ & 0.96 & 2.77 & $3.576(6)$ & 141 \\
\hline
\end{tabular}

Symmetry code: (i) $-x+2,-y-1,-z+1$.

central enone group is confirmed by the $\mathrm{C} 5-\mathrm{C} 6=\mathrm{C} 7-\mathrm{C} 8$ torsion angle of $178.3(4)^{\circ}$. The carbonyl group at $\mathrm{C} 5$ lies in the plane of the olefinic double bond and chloro-thiophene rings as indicated by the $\mathrm{O} 1-\mathrm{C} 5-\mathrm{C} 4-\mathrm{C} 3$ and $\mathrm{O} 1-\mathrm{C} 5-\mathrm{C} 6-\mathrm{C} 7$ torsion angle values of $177.9(5)^{\circ}$ and $-11.7(6)^{\circ}$, respectively.

In the crystal, molecules are linked by pairs of $\mathrm{C}-\mathrm{H} \cdots \pi$ interactions, forming inversion dimers (Table 1 and Fig. 2). There are no other significant intermolecular interactions present.

\section{Synthesis and crystallization}

A mixture of 4-methylbenzaldehyde (5 mmol), 5-chloro-2acetylthiophene $(5 \mathrm{mmol})$ and sodium hydroxide $(5 \mathrm{mmol})$ in $95 \%$ ethyl alcohol $(25 \mathrm{ml})$ was stirred at room temperature for $3 \mathrm{~h}$. The progress of the reaction was monitored by TLC. After completion of the reaction, the mixture was poured into ice-

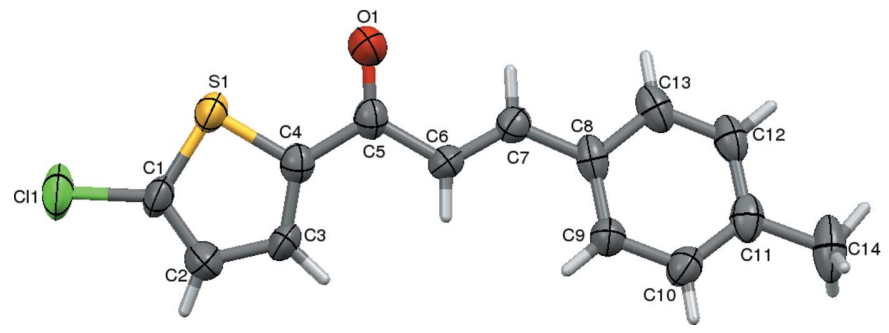

Figure 1

The molecular structure of the title compound, showing the atom labelling. Displacement ellipsoids are drawn at the $50 \%$ probability level.

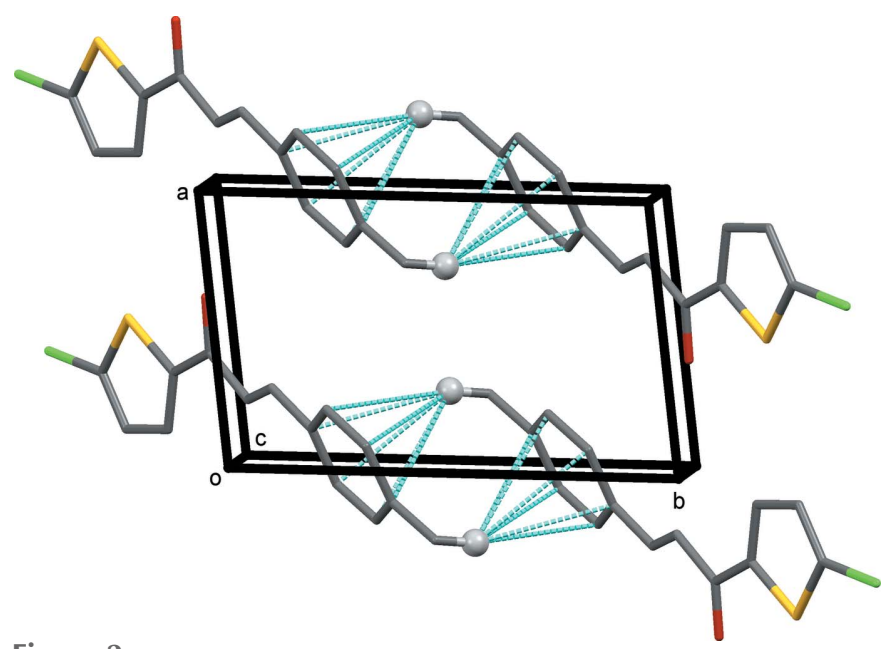

Figure 2

A view along the $c$ axis of the crystal packing of the title compound. The $\mathrm{C}-\mathrm{H} \cdots \pi$ interactions (Table 1 ) are represented by dashed lines, and only $\mathrm{H}$ atom $\mathrm{H} 14 A$ (grey ball) has been included.

Table 2

Experimental details.

Crystal data

Chemical formula

$M_{\mathrm{r}}$

Crystal system, space group

Temperature (K)

$a, b, c(\AA)$

$\alpha, \beta, \gamma\left({ }^{\circ}\right)$

$V\left(\AA^{3}\right)$

$Z$

Radiation type

$\mu\left(\mathrm{mm}^{-1}\right)$

Crystal size $(\mathrm{mm})$

Data collection

Diffractometer

Absorption correction

$T_{\min }, T_{\max }$

No. of measured, independent and observed $[I>2 \sigma(I)]$ reflections

$R_{\text {int }}$

$(\sin \theta / \lambda)_{\max }\left(\AA^{-1}\right)$

Refinement

$R\left[F^{2}>2 \sigma\left(F^{2}\right)\right], w R\left(F^{2}\right), S$

No. of reflections

No. of parameters

$\mathrm{H}$-atom treatment

$\Delta \rho_{\max }, \Delta \rho_{\min }\left(\mathrm{e} \AA^{-3}\right)$

Computer programs: CrystalClear (Rigaku, 2011), SHELXS97and SHELXL97 (Sheldrick, 2008) and Mercury (Macrae et al., 2008).

cold water and kept in the refrigerator overnight. The solid formed was filtered, and washed with cold hydrochloric acid (5\%). Block-like colourless crystals of the title compound were obtained by crystallization from methanol by the slow evaporation technique (yield 84\%, m.p. 403-405 K).

\section{Refinement}

Crystal data, data collection and structure refinement details are summarized in Table 2.

\section{Acknowledgements}

The authors are grateful to the National Single Crystal X-ray Diffractometer facility, Department of Studies in Physics, University of Mysore, India, for providing the X-ray intensity data.

\section{References}

Macrae, C. F., Bruno, I. J., Chisholm, J. A., Edgington, P. R., McCabe, P., Pidcock, E., Rodriguez-Monge, L., Taylor, R., van de Streek, J. \& Wood, P. A. (2008). J. Appl. Cryst. 41, 466-470.

Naveen, S., Dileep Kumar, A., Ajay Kumar, K., Manjunath, H. R., Lokanath, N. K. \& Warad, I. (2016a). IUCrData, 1, x161800.

Naveen, S., Prabhudeva, M. G., Ajay Kumar, K., Lokanath, N. K. \& Abdoh, M. (2016b). IUCrData, 1, x161974.

Rigaku. (1999). NUMABS. Rigaku Corporation, Tokyo, Japan.

Rigaku. (2011). CrystalClear. Rigaku Corporation, Tokyo, Japan.

Sheldrick, G. M. (2008). Acta Cryst. A64, 112-122.

Tejkiran, P. J., Brahma Teja, M. S., Sai Siva Kumar, P., Sankar, P., Philip, R., Naveen, S., Lokanath, N. K. \& Nageswara Rao, G. (2016). J. Photochem. Photobiol. Chem. A, 324, 33-39. 


\section{full crystallographic data}

IUCrData (2017). 2, x170038 [https://doi.org/10.1107/S2414314617000384]

(E)-1-(5-Chlorothiophen-2-yl)-3-(p-tolyl)prop-2-en-1-one

Karthik Kumara, S. Naveen, M. G. Prabhudeva, K. Ajay Kumar, N. K. Lokanath and Ismail Warad

(E)-1-(5-Chlorothiophen-2-yl)-3-(p-tolyl) prop-2-en-1-one

Crystal data

$\mathrm{C}_{14} \mathrm{H}_{11} \mathrm{ClOS}$

$M_{r}=262.75$

Triclinic, $P \overline{1}$

Hall symbol: -P 1

$a=6.000(2) \AA$

$b=9.718(4) \AA$

$c=10.936(4) \AA$

$\alpha=94.268(17)^{\circ}$

$\beta=93.83(3)^{\circ}$

$\gamma=96.97(2)^{\circ}$

$V=629.3(4) \AA^{3}$

$$
Z=2
$$

$F(000)=272$

$D_{\mathrm{x}}=1.387 \mathrm{Mg} \mathrm{m}^{-3}$

Mo $K \alpha$ radiation, $\lambda=0.71073 \AA$

Cell parameters from 3635 reflections

$\theta=3.4-27.6^{\circ}$

$\mu=0.45 \mathrm{~mm}^{-1}$

$T=293 \mathrm{~K}$

Block, colourless

$0.39 \times 0.31 \times 0.27 \mathrm{~mm}$

\section{Data collection}

Rigaku Saturn724+

diffractometer

Radiation source: fine-focus sealed tube

Graphite monochromator

Detector resolution: 18.4 pixels $\mathrm{mm}^{-1}$

profile data from $\omega$-scans

Absorption correction: multi-scan

(NUMABS; Rigaku, 1999)

$T_{\min }=0.972, T_{\max }=0.976$

3635 measured reflections

2808 independent reflections

1279 reflections with $I>2 \sigma(I)$

$R_{\text {int }}=0.071$

$\theta_{\max }=27.6^{\circ}, \theta_{\min }=3.4^{\circ}$

$h=-7 \rightarrow 7$

$k=-11 \rightarrow 12$

$l=-13 \rightarrow 14$

Refinement

Refinement on $F^{2}$

Least-squares matrix: full

Secondary atom site location: difference Fourier map

$R\left[F^{2}>2 \sigma\left(F^{2}\right)\right]=0.067$

$w R\left(F^{2}\right)=0.264$

$S=0.90$

2808 reflections

155 parameters

0 restraints

Primary atom site location: structure-invariant direct methods

Hydrogen site location: inferred from neighbouring sites

$\mathrm{H}$-atom parameters constrained

$w=1 /\left[\sigma^{2}\left(F_{\mathrm{o}}{ }^{2}\right)+(0.1466 P)^{2}\right]$

where $P=\left(F_{\mathrm{o}}{ }^{2}+2 F_{\mathrm{c}}{ }^{2}\right) / 3$

$(\Delta / \sigma)_{\max }<0.001$

$\Delta \rho_{\max }=0.51$ e $\AA^{-3}$

$\Delta \rho_{\min }=-0.65$ e $\AA^{-3}$

Special details

Geometry. Bond distances, angles etc. have been calculated using the rounded fractional coordinates. All su's are estimated from the variances of the (full) variance-covariance matrix. The cell esds are taken into account in the estimation of distances, angles and torsion angles 
Refinement. Refinement on $\mathrm{F}^{2}$ for ALL reflections except those flagged by the user for potential systematic errors. Weighted R-factors $\mathrm{wR}$ and all goodnesses of fit $\mathrm{S}$ are based on $\mathrm{F}^{2}$, conventional R-factors $\mathrm{R}$ are based on $\mathrm{F}$, with $\mathrm{F}$ set to zero for negative $\mathrm{F}^{2}$. The observed criterion of $\mathrm{F}^{2}>2 \operatorname{sigma}\left(\mathrm{F}^{2}\right)$ is used only for calculating -R-factor-obs etc. and is not relevant to the choice of reflections for refinement. $\mathrm{R}$-factors based on $\mathrm{F}^{2}$ are statistically about twice as large as those based on F, and R-factors based on ALL data will be even larger.

Fractional atomic coordinates and isotropic or equivalent isotropic displacement parameters $\left(\AA^{2}\right)$

\begin{tabular}{|c|c|c|c|c|}
\hline & $x$ & $y$ & $z$ & $U_{\text {iso }} * / U_{\text {eq }}$ \\
\hline $\mathrm{Cl1}$ & $0.6471(3)$ & $0.42181(15)$ & $-0.15570(14)$ & $0.0791(6)$ \\
\hline $\mathrm{S} 1$ & $0.5125(2)$ & $0.22896(12)$ & $0.03032(11)$ & $0.0490(4)$ \\
\hline $\mathrm{O} 1$ & $0.4218(6)$ & $0.0428(4)$ & $0.2208(3)$ & 0.0609 (12) \\
\hline $\mathrm{C} 1$ & $0.7155(8)$ & $0.3040(5)$ & -0.0540 & 0.0473 (16) \\
\hline $\mathrm{C} 2$ & $0.9174(9)$ & $0.2628(5)$ & $-0.0289(5)$ & $0.0566(17)$ \\
\hline C3 & $0.9106(8)$ & $0.1657(5)$ & 0.0608 (4) & $0.0494(17)$ \\
\hline $\mathrm{C} 4$ & $0.7015(7)$ & $0.1373(4)$ & $0.1020(4)$ & $0.0421(14)$ \\
\hline $\mathrm{C} 5$ & $0.6217(8)$ & 0.0486 (4) & $0.1973(4)$ & $0.0435(14)$ \\
\hline C6 & $0.7863(7)$ & $-0.0282(4)$ & $0.2624(4)$ & $0.0415(12)$ \\
\hline $\mathrm{C} 7$ & $0.7370(8)$ & $-0.0886(4)$ & $0.3637(4)$ & $0.0423(12)$ \\
\hline $\mathrm{C} 8$ & $0.8780(9)$ & -0.1707 (4) & $0.4362(4)$ & 0.0463 (14) \\
\hline $\mathrm{C} 9$ & $1.0772(8)$ & $-0.2111(5)$ & $0.3982(4)$ & 0.0475 (17) \\
\hline $\mathrm{C} 10$ & $1.2012(8)$ & $-0.2947(5)$ & $0.4676(4)$ & 0.0499 (17) \\
\hline C11 & $1.1293(9)$ & $-0.3381(4)$ & $0.5784(4)$ & 0.0485 (16) \\
\hline $\mathrm{C} 12$ & $0.9276(9)$ & $-0.2947(5)$ & $0.6170(4)$ & $0.0516(16)$ \\
\hline $\mathrm{C} 13$ & $0.8017(9)$ & $-0.2132(5)$ & $0.5487(4)$ & 0.0525 (14) \\
\hline C14 & $1.2604(11)$ & $-0.4281(5)$ & $0.6534(5)$ & $0.075(2)$ \\
\hline $\mathrm{H} 2$ & 1.04670 & 0.29450 & -0.06610 & $0.0680 *$ \\
\hline H3 & 1.03520 & 0.12540 & 0.08890 & $0.0600 *$ \\
\hline H6 & 0.92600 & -0.03400 & 0.23170 & $0.0500 *$ \\
\hline H7 & 0.59690 & -0.07760 & 0.39200 & $0.0510^{*}$ \\
\hline H9 & 1.12970 & -0.18200 & 0.32510 & $0.0570^{*}$ \\
\hline H10 & 1.33340 & -0.32170 & 0.43930 & $0.0600 *$ \\
\hline H12 & 0.87730 & -0.32190 & 0.69120 & $0.0620^{*}$ \\
\hline H13 & 0.66870 & -0.18680 & 0.57640 & $0.0630^{*}$ \\
\hline H14A & 1.26880 & -0.51450 & 0.60630 & $0.1130^{*}$ \\
\hline H14B & 1.18680 & -0.44620 & 0.72680 & $0.1130^{*}$ \\
\hline $\mathrm{H} 14 \mathrm{C}$ & 1.40970 & -0.38140 & 0.67500 & $0.1130 *$ \\
\hline
\end{tabular}

Atomic displacement parameters $\left(\AA^{2}\right)$

\begin{tabular}{lllllll}
\hline & $U^{11}$ & $U^{22}$ & $U^{33}$ & $U^{12}$ & $U^{13}$ & $U^{23}$ \\
\hline C11 & $0.1032(13)$ & $0.0772(10)$ & $0.0688(10)$ & $0.0388(9)$ & $0.0066(9)$ & $0.0402(8)$ \\
S1 & $0.0518(8)$ & $0.0535(7)$ & $0.0465(7)$ & $0.0221(6)$ & $0.0015(6)$ & $0.0126(5)$ \\
O1 & $0.063(2)$ & $0.071(2)$ & $0.057(2)$ & $0.0232(19)$ & $0.0186(19)$ & $0.0236(18)$ \\
C1 & $0.054(3)$ & $0.047(2)$ & $0.046(3)$ & $0.024(2)$ & $0.000(2)$ & $0.013(2)$ \\
C2 & $0.048(3)$ & $0.066(3)$ & $0.060(3)$ & $0.009(3)$ & $0.011(2)$ & $0.024(3)$ \\
C3 & $0.045(3)$ & $0.056(3)$ & $0.054(3)$ & $0.025(2)$ & $0.005(2)$ & $0.019(2)$ \\
C4 & $0.050(3)$ & $0.040(2)$ & $0.037(2)$ & $0.009(2)$ & $0.002(2)$ & $0.0042(18)$
\end{tabular}




\begin{tabular}{lllllll} 
C5 & $0.055(3)$ & $0.041(2)$ & $0.036(2)$ & $0.011(2)$ & $0.003(2)$ & $0.0051(18)$ \\
C6 & $0.035(2)$ & $0.047(2)$ & $0.045(2)$ & $0.0122(19)$ & $0.004(2)$ & $0.009(2)$ \\
C7 & $0.041(2)$ & $0.041(2)$ & $0.046(2)$ & $0.0092(19)$ & $0.002(2)$ & $0.0063(19)$ \\
C8 & $0.069(3)$ & $0.034(2)$ & $0.034(2)$ & $0.000(2)$ & $-0.002(2)$ & $0.0073(17)$ \\
C9 & $0.049(3)$ & $0.052(3)$ & $0.045(3)$ & $0.014(2)$ & $0.006(2)$ & $0.013(2)$ \\
C10 & $0.046(3)$ & $0.052(3)$ & $0.053(3)$ & $0.013(2)$ & $-0.003(2)$ & $0.009(2)$ \\
C11 & $0.061(3)$ & $0.036(2)$ & $0.045(3)$ & $-0.002(2)$ & $-0.011(2)$ & $0.0065(19)$ \\
C12 & $0.065(3)$ & $0.050(3)$ & $0.037(2)$ & $-0.004(2)$ & $-0.005(2)$ & $0.012(2)$ \\
C13 & $0.069(3)$ & $0.048(2)$ & $0.038(2)$ & $-0.005(2)$ & $0.004(2)$ & $0.008(2)$ \\
C14 & $0.115(5)$ & $0.048(3)$ & $0.056(3)$ & $-0.006(3)$ & $-0.031(3)$ & $0.018(2)$ \\
\hline
\end{tabular}

Geometric parameters $\left(\AA,{ }^{\circ}\right)$

\begin{tabular}{|c|c|c|c|}
\hline $\mathrm{C} 11-\mathrm{C} 1$ & $1.720(5)$ & $\mathrm{C} 11-\mathrm{C} 12$ & $1.409(7)$ \\
\hline $\mathrm{S} 1-\mathrm{C} 1$ & $1.703(5)$ & $\mathrm{C} 11-\mathrm{C} 14$ & $1.498(7)$ \\
\hline $\mathrm{S} 1-\mathrm{C} 4$ & $1.710(4)$ & $\mathrm{C} 12-\mathrm{C} 13$ & $1.383(7)$ \\
\hline $\mathrm{O} 1-\mathrm{C} 5$ & $1.239(6)$ & $\mathrm{C} 2-\mathrm{H} 2$ & 0.9300 \\
\hline $\mathrm{C} 1-\mathrm{C} 2$ & $1.339(7)$ & $\mathrm{C} 3-\mathrm{H} 3$ & 0.9300 \\
\hline $\mathrm{C} 2-\mathrm{C} 3$ & $1.410(7)$ & $\mathrm{C} 6-\mathrm{H} 6$ & 0.9300 \\
\hline $\mathrm{C} 3-\mathrm{C} 4$ & $1.365(6)$ & $\mathrm{C} 7-\mathrm{H} 7$ & 0.9300 \\
\hline $\mathrm{C} 4-\mathrm{C} 5$ & $1.469(6)$ & C9-H9 & 0.9300 \\
\hline $\mathrm{C} 5-\mathrm{C} 6$ & $1.484(6)$ & $\mathrm{C} 10-\mathrm{H} 10$ & 0.9300 \\
\hline $\mathrm{C} 6-\mathrm{C} 7$ & $1.326(6)$ & $\mathrm{C} 12-\mathrm{H} 12$ & 0.9300 \\
\hline $\mathrm{C} 7-\mathrm{C} 8$ & $1.466(6)$ & $\mathrm{C} 13-\mathrm{H} 13$ & 0.9300 \\
\hline $\mathrm{C} 8-\mathrm{C} 9$ & $1.383(7)$ & $\mathrm{C} 14-\mathrm{H} 14 \mathrm{~A}$ & 0.9600 \\
\hline $\mathrm{C} 8-\mathrm{C} 13$ & $1.414(6)$ & C14-H14B & 0.9600 \\
\hline $\mathrm{C} 9-\mathrm{C} 10$ & $1.397(7)$ & $\mathrm{C} 14-\mathrm{H} 14 \mathrm{C}$ & 0.9600 \\
\hline $\mathrm{C} 10-\mathrm{C} 11$ & $1.391(6)$ & & \\
\hline $\mathrm{C} 1-\mathrm{S} 1-\mathrm{C} 4$ & $91.1(2)$ & $\mathrm{C} 8-\mathrm{C} 13-\mathrm{C} 12$ & $119.1(5)$ \\
\hline $\mathrm{C} 11-\mathrm{C} 1-\mathrm{S} 1$ & $119.3(3)$ & $\mathrm{C} 1-\mathrm{C} 2-\mathrm{H} 2$ & 124.00 \\
\hline $\mathrm{C} 11-\mathrm{C} 1-\mathrm{C} 2$ & $127.7(4)$ & $\mathrm{C} 3-\mathrm{C} 2-\mathrm{H} 2$ & 124.00 \\
\hline $\mathrm{S} 1-\mathrm{C} 1-\mathrm{C} 2$ & $113.0(4)$ & $\mathrm{C} 2-\mathrm{C} 3-\mathrm{H} 3$ & 124.00 \\
\hline $\mathrm{C} 1-\mathrm{C} 2-\mathrm{C} 3$ & $111.9(5)$ & $\mathrm{C} 4-\mathrm{C} 3-\mathrm{H} 3$ & 124.00 \\
\hline $\mathrm{C} 2-\mathrm{C} 3-\mathrm{C} 4$ & $112.7(4)$ & $\mathrm{C} 5-\mathrm{C} 6-\mathrm{H} 6$ & 120.00 \\
\hline $\mathrm{S} 1-\mathrm{C} 4-\mathrm{C} 3$ & $111.2(3)$ & $\mathrm{C} 7-\mathrm{C} 6-\mathrm{H} 6$ & 120.00 \\
\hline $\mathrm{S} 1-\mathrm{C} 4-\mathrm{C} 5$ & $117.9(3)$ & $\mathrm{C} 6-\mathrm{C} 7-\mathrm{H} 7$ & 116.00 \\
\hline $\mathrm{C} 3-\mathrm{C} 4-\mathrm{C} 5$ & $130.9(4)$ & $\mathrm{C} 8-\mathrm{C} 7-\mathrm{H} 7$ & 116.00 \\
\hline $\mathrm{O} 1-\mathrm{C} 5-\mathrm{C} 4$ & $118.7(4)$ & $\mathrm{C} 8-\mathrm{C} 9-\mathrm{H} 9$ & 119.00 \\
\hline $\mathrm{O} 1-\mathrm{C} 5-\mathrm{C} 6$ & $123.1(4)$ & $\mathrm{C} 10-\mathrm{C} 9-\mathrm{H} 9$ & 119.00 \\
\hline $\mathrm{C} 4-\mathrm{C} 5-\mathrm{C} 6$ & $118.2(4)$ & $\mathrm{C} 9-\mathrm{C} 10-\mathrm{H} 10$ & 120.00 \\
\hline $\mathrm{C} 5-\mathrm{C} 6-\mathrm{C} 7$ & $120.9(4)$ & $\mathrm{C} 11-\mathrm{C} 10-\mathrm{H} 10$ & 120.00 \\
\hline $\mathrm{C} 6-\mathrm{C} 7-\mathrm{C} 8$ & $127.4(4)$ & $\mathrm{C} 11-\mathrm{C} 12-\mathrm{H} 12$ & 119.00 \\
\hline $\mathrm{C} 7-\mathrm{C} 8-\mathrm{C} 9$ & $123.2(4)$ & $\mathrm{C} 13-\mathrm{C} 12-\mathrm{H} 12$ & 119.00 \\
\hline $\mathrm{C} 7-\mathrm{C} 8-\mathrm{C} 13$ & $117.8(5)$ & $\mathrm{C} 8-\mathrm{C} 13-\mathrm{H} 13$ & 120.00 \\
\hline $\mathrm{C} 9-\mathrm{C} 8-\mathrm{C} 13$ & $119.0(4)$ & $\mathrm{C} 12-\mathrm{C} 13-\mathrm{H} 13$ & 120.00 \\
\hline $\mathrm{C} 8-\mathrm{C} 9-\mathrm{C} 10$ & $121.3(4)$ & $\mathrm{C} 11-\mathrm{C} 14-\mathrm{H} 14 \mathrm{~A}$ & 109.00 \\
\hline $\mathrm{C} 9-\mathrm{C} 10-\mathrm{C} 11$ & $120.8(4)$ & $\mathrm{C} 11-\mathrm{C} 14-\mathrm{H} 14 \mathrm{~B}$ & 110.00 \\
\hline
\end{tabular}




$\begin{array}{llll}\mathrm{C} 10-\mathrm{C} 11-\mathrm{C} 12 & 117.4(4) & \mathrm{C} 11-\mathrm{C} 14-\mathrm{H} 14 \mathrm{C} & 109.00 \\ \mathrm{C} 10-\mathrm{C} 11-\mathrm{C} 14 & 121.3(5) & \mathrm{H} 14 \mathrm{~A}-\mathrm{C} 14-\mathrm{H} 14 \mathrm{~B} & 109.00 \\ \mathrm{C} 12-\mathrm{C} 11-\mathrm{C} 14 & 121.3(4) & \mathrm{H} 14 \mathrm{~A}-\mathrm{C} 14-\mathrm{H} 14 \mathrm{C} & 109.00 \\ \mathrm{C} 11-\mathrm{C} 12-\mathrm{C} 13 & 122.5(4) & \mathrm{H} 14 \mathrm{~B}-\mathrm{C} 14-\mathrm{H} 14 \mathrm{C} & 110.00 \\ & & & \\ \mathrm{C} 4-\mathrm{S} 1-\mathrm{C} 1-\mathrm{C} 11 & -179.2(3) & \mathrm{C} 4-\mathrm{C} 5-\mathrm{C} 6-\mathrm{C} 7 & 167.3(4) \\ \mathrm{C} 4-\mathrm{S} 1-\mathrm{C} 1-\mathrm{C} 2 & -0.4(4) & \mathrm{C} 5-\mathrm{C} 6-\mathrm{C} 7-\mathrm{C} 8 & 178.3(4) \\ \mathrm{C} 1-\mathrm{S} 1-\mathrm{C} 4-\mathrm{C} 3 & 0.1(4) & \mathrm{C} 6-\mathrm{C} 7-\mathrm{C} 8-\mathrm{C} 9 & -8.4(7) \\ \mathrm{C} 1-\mathrm{S} 1-\mathrm{C} 4-\mathrm{C} 5 & 177.8(3) & \mathrm{C} 6-\mathrm{C} 7-\mathrm{C} 8-\mathrm{C} 13 & 173.4(4) \\ \mathrm{C} 11-\mathrm{C} 1-\mathrm{C} 2-\mathrm{C} 3 & 179.3(4) & \mathrm{C} 7-\mathrm{C} 8-\mathrm{C} 9-\mathrm{C} 10 & -177.1(4) \\ \mathrm{S} 1-\mathrm{C} 1-\mathrm{C} 2-\mathrm{C} 3 & 0.7(6) & \mathrm{C} 13-\mathrm{C} 8-\mathrm{C} 9-\mathrm{C} 10 & 1.2(7) \\ \mathrm{C} 1-\mathrm{C} 2-\mathrm{C} 3-\mathrm{C} 4 & -0.6(6) & \mathrm{C} 7-\mathrm{C} 8-\mathrm{C} 13-\mathrm{C} 12 & 177.9(4) \\ \mathrm{C} 2-\mathrm{C} 3-\mathrm{C} 4-\mathrm{S} 1 & 0.3(5) & \mathrm{C} 9-\mathrm{C} 8-\mathrm{C} 13-\mathrm{C} 12 & -0.5(7) \\ \mathrm{C} 2-\mathrm{C} 3-\mathrm{C} 4-\mathrm{C} 5 & -177.0(4) & \mathrm{C} 8-\mathrm{C} 9-\mathrm{C} 10-\mathrm{C} 11 & -1.0(7) \\ \mathrm{S} 1-\mathrm{C} 4-\mathrm{C} 5-\mathrm{O} 1 & 0.7(5) & \mathrm{C} 9-\mathrm{C} 10-\mathrm{C} 11-\mathrm{C} 12 & 0.1(7) \\ \mathrm{S} 1-\mathrm{C} 4-\mathrm{C} 5-\mathrm{C} 6 & -178.3(3) & \mathrm{C} 9-\mathrm{C} 10-\mathrm{C} 11-\mathrm{C} 14 & 179.8(4) \\ \mathrm{C} 3-\mathrm{C} 4-\mathrm{C} 5-\mathrm{O} 1 & 177.9(5) & \mathrm{C} 10-\mathrm{C} 11-\mathrm{C} 12-\mathrm{C} 13 & 0.6(7) \\ \mathrm{C} 3-\mathrm{C} 4-\mathrm{C} 5-\mathrm{C} 6 & -1.1(7) & \mathrm{C} 14-\mathrm{C} 11-\mathrm{C} 12-\mathrm{C} 13 & -179.1(5) \\ \mathrm{O} 1-\mathrm{C} 5-\mathrm{C} 6-\mathrm{C} 7 & -11.7(6) & \mathrm{C} 11-\mathrm{C} 12-\mathrm{C} 13-\mathrm{C} 8 & -0.4(7)\end{array}$

Hydrogen-bond geometry $\left(\AA,{ }^{\circ}\right)$

$\mathrm{Cg}$ is the centroid of the $\mathrm{C} 8-\mathrm{C} 13$ ring.

\begin{tabular}{lllll}
\hline$D-\mathrm{H} \cdots A$ & $D-\mathrm{H}$ & $\mathrm{H} \cdots A$ & $D \cdots A$ & $D-\mathrm{H} \cdots A$ \\
\hline $\mathrm{C} 14-\mathrm{H} 14 A \cdots C g^{\mathrm{i}}$ & 0.96 & 2.77 & $3.576(6)$ & 141 \\
\hline
\end{tabular}

Symmetry code: (i) $-x+2,-y-1,-z+1$. 